\title{
Carbon Nanotube Fibers Prepared by Activating Deactivated Iron Particles in Floating Catalyst Chemical Vapor Deposition Tail Gas
}

\author{
Yancai ZHANG*, Shuaishuai HAN \\ Material Science and Engineering, Tianjin university, Weijin road, Nankai district, Tianjin city, China \\ crossref http://dx.doi.org/10.5755/j01.ms.23.3.17050
}

Received 29 November 2016; accepted 08 January 2017

\begin{abstract}
Catalysts can determine the structure and properties of carbon nanotube (CNT) fibers fabricated using the floating catalyst chemical vapor deposition (FCCVD) method. The tail gas left over when CNT fibers are fabricated by the FCCVD method has been proven to contain deactivated iron nanoparticles, as well as carbide gas and hydrogen. This study demonstrates that the deactivated iron nanoparticles in tail gas can be successfully activated in a double furnace system under certain conditions. CNT fibers can be successfully prepared using the activated iron nanoparticles by adding the precursor without the catalyst. These CNT fibers are composed of double-walled carbon nanotubes (DWNTs) and have low density, high strength, and electrical conductivity.

Keywords: activating iron particles, reclaiming tail gas, CNT fibers.
\end{abstract}

\section{INTRODUCTION}

Carbon nanotubes (CNTs) are characterized by a typical nanostructure along with extraordinary mechanical, electrical, and thermal properties [1-3]. These features and their other functionalities make CNTs suitable for applications as structural and functional materials, including composites [4-7], conductors [8-10], catalyst supports [11, 12], and sensors [13, 14]. CNT fiber is macroscopic assembly of carbon nanotubes, formed with CNTs alignment along the axial direction. Because of weak links, deficiencies, and impurities in the scattering of CNT assemblies, the properties of CNT fibers are worse than ones of single CNT, but CNT fibers can compete with other fibers in high-end uses, especially in applications requiring combined electrical and mechanical functionalities. Therefore, the large-scale production of CNT fibers is important target of research and development [15].

Several processes have been used to fabricate macroscopic CNT fibers, including wet spinning of CNT yarns [16-19], dry spinning of CNT yarns [20-23], and direct spinning of continuous fibers from CNT aerogel by Floating Catalyst Chemical Vapor Deposition (FCCVD) [24-27]. FCCVD is the irreplaceable method of fabricating CNT fibers, because it can meet large-scale commercial demand [28]. Furthermore, the strength of CNT fibers can reach $5.53 \mathrm{GPa}$ [29], and the structure of CNT fibers can be controlled [30]. Because of its high degree of controllability and scalability, CVD is currently the most widely applied method for preparing CNT fibers.

Catalysts can determine the structure of CNT fibers fabricated through the FCCVD method, affecting the fibers' length, walls, and chirality. CNT fibers with different structures and performances, such as singlewalled carbon nanotube (SWNT), DWNT, multi-walled carbon nanotube (MWNT) fibers [30,31], ultra-pure CNT

\footnotetext{
${ }^{*}$ Corresponding author. Tel.: +8610-18302896296.

E-mail address: zhangyancai@tju.edu.cn (Y. Zhang)
}

fibers [32], and metallic chirality SWNT fibers [33], can be fabricated by adjusting the morphology of the catalysts.

However, tail gas of CNT fibers prepared by the FCCVD method has been proven to contain deactivated iron particles, except for hydrogen and carbide gas [34]. The tail gas is directly injected into the air after simple filtration. Reclaiming tail gas represents a significant advance in fabricating CNT fibers because it can save resources, protect the environment, and promote the largescale production of CNT fibers. In this study, FCCVD tail gas was collected and analyzed in order to study the morphology of deactivated iron particles in tail gas. First, a double furnace system was first designed to fabricate CNT fibers while reclaiming tail gas. Different methods were developed to activate the iron particles. Then, the structure and properties of CNT fibers prepared by activating iron particles in tail gas were studied. Preparing CNT fibers by reclaiming tail gas is beneficial for studying the effect of catalysts on the synthesis of CNT fibers by FCCVD, promoting the development of CNT fibers, saving resources, and protecting the environment.

\section{EXPERIMENTAL METHODS}

\subsection{Double furnace system}

This study applied the same FCCVD method and spinning process used by Zhong [28] to fabricate CNT fibers. This method involves connecting two vertical furnaces of spinning CNT fibers using pipelines (double furnaces system, as shown in Fig. 1) to fabricate CNT fibers by reclaiming CVD tail gas and activating iron nanoparticles. The primary components of the double furnace system are two furnaces (a front furnace and a rear furnace), two spinning systems, and one set of pipelines that can induce the tail gas of the front furnace to flow into rear furnace. The pipelines were used to connect the outlet of the front furnace and the inlet of the rear furnace. A flowmeter was placed in the middle to calculate and control the quantity of tail gas flowing into the rear 
furnace.

Before reclaiming tail gas from the CNT fibers fabricated by CVD, the front furnace should normally spin CNT fibers (FCNT fibers) by adjusting conditions like the location of the end of needle, the rate of hydrogen flow, and the injecting rate of feedstock $[32,35]$. Then, the rear furnace prepares CNT fibers (RCNT fibers) by reclaiming tail gas from the front furnace.

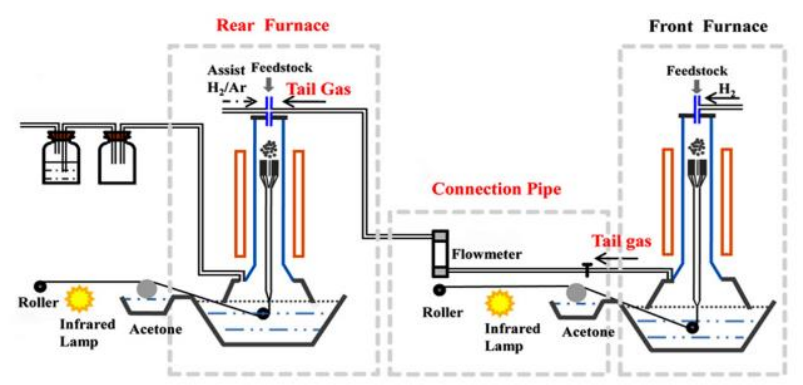

Fig. 1. Schematic diagram of the double furnace system

Unlike single furnace was developed over a decade ago $[26,28]$, the pressure of the front furnace in the double furnace system gradually increases due to the rear furnace, such that the precursor can not be injected into the reactor to form aerogel continuously. Even though the sock-like CNT assembly was in good condition, the assembly could not arrive to the reactor bottom with flow. In order to reduce pressure in the front furnace, a flowmeter was introduced to control the rate of tail gas flowing into the rear furnace and a pump was added to control the pressure of the front furnace.

\subsection{Experimental procedures}

The temperatures of the both furnaces were raised at the same time at rate of $0.2{ }^{\circ} \mathrm{C} \mathrm{s}^{-1}$. Meanwhile, argon flow was introduced into both reactors at rate of $200 \mathrm{sccm}$. When the temperature in both reactors reached $1170{ }^{\circ} \mathrm{C}$, hydrogen flow was introduced into the front reactor at a rate of $1000 \mathrm{sccm}$. Hydrocarbon feedstock was injected into the front reactor with a syringe pump at rate of 8 to

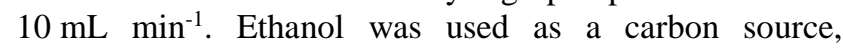
dispersed with ferrocene $(2.4 \mathrm{wt} . \%)$ as a catalyst and thiophene $(0.6 \mathrm{wt} . \%)$ as a promoter. After the front furnace began normally spinning FCNT fibers, CVD tail gas from the front furnace was poured into the rear reactor at a rate of 700 to $800 \mathrm{sccm}$ through the pipeline, with the quantity of tail gas being detected by the flowmeter. Furthermore, the CVD tail gas was collected by bladder, and gas chromatography (GC) was used to analyze all the components of the tail gas. The outlet hole of the tail gas bottle was wrapped by copper grid with 120 meshes per inch to collect particles in the tail gas. The particles were detected using scanning electron microscopy (SEM) and transmission electron microscopy (TEM).

Different methods were designed to activate unused iron nanoparticles in the tail gas. During the FCCVD process, catalysts can become inactive due to the accumulation of amorphous carbon. In order to activate the inactive iron particles, the amorphous carbon layer must first be removed. We used the characteristics of carbon layer reactions with hydrogen and water vapor at high temperatures to activate the deactivated iron particles. Before the tail gas was introduced into the rear furnace reactor, deactivated iron particles flowed through the pipelines with the tail gas. After the rear furnace was purged with flowing tail gas for approximately $10 \mathrm{~min}$, the precursor without catalyst and deionized water were injected into the reactor with a syringe pump. Ethanol was used as carbon source, dispersed with thiophene as promoter agents. The precursor was injected at rate of 6 to $8 \mathrm{~mL} \mathrm{~min}^{-1}$, and the water was injected at rate of 2 to $3 \mathrm{~mL} \mathrm{~min}^{-1}$. Then, other conditions were adjusted to activate iron particles in tail gas to fabricate CNT fibers.

\subsection{Assessing the performance and structure of CNT yarns}

The diameter and surface of the fibers were analyzed with an optical microscope (U-25LBD, Olympus, Japan). The morphology of the fibers was observed under SEM (JSP-6700F, Philips, Holland) by attaching a slice of fiber on a copper plate. The CNT structure of the fibers was studied through TEM (Tecnai-G2 F20, Philips, Holland) by placing a piece of fiber on a copper grid. Defects in the fibers were analyzed under Raman spectroscopy (Invia Reflex, Renishaw, UK) by attaching a length of fiber to a glass slide, the Raman wavelength was $532 \mathrm{~nm}$. Mechanical properties were studied using a micro tensile tester (Microfiber Tester XQ-1, Shanghai Co., China) and a $10 \mathrm{~mm}$ fiber sample at tensile speed of $3 \mathrm{~mm} \mathrm{~min}^{-1}$. Electrical conductivity was measured using two-pole method with a multimeter. The components of the CVD tail gas were analyzed by SEM, TEM, and GC (GC2010, Shimadzu, Japan).

\section{RESULTS AND DISCUSSION}

First, the components of FCCVD tail gas were first analyzed. According GC results, the tail gas was $12.78 \mathrm{~mol} \%$ methane, $8.53 \mathrm{~mol} \%$ carbon monoxide, $0.08 \mathrm{~mol} \%$ carbon dioxide, and $78.60 \mathrm{~mol} \%$ hydrogen. Ethylene and hydrogen sulphide were also detected at negligible quantities.

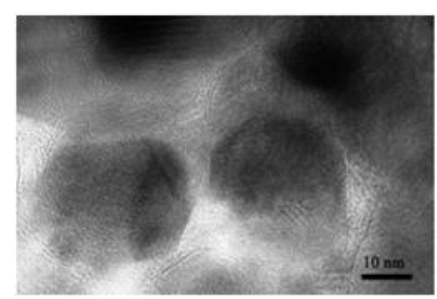

a

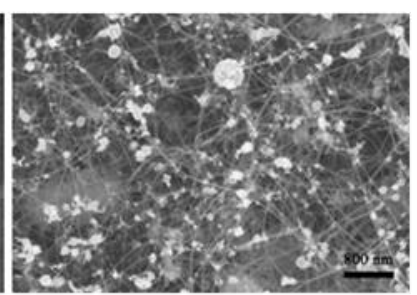

b
Fig. 2. The structure of particles in the tail gas: a-TEM of the deactivated iron nanoparticles; $b-$ SEM of the particles

The particles in tail gas were CNTs and deactivated iron nanoparticles according to Fig. 2. The deactivated iron particles in the tail gas were wrapped by amorphous carbon and graphite layers, the diameter of the iron particles was in range of $20 \mathrm{~nm}$ to $30 \mathrm{~nm}$. This result indicates that FCCVD tail gas contains not only carbide gas and hydrogen, but also CNTs and deactivated iron nanoparticles. 
CNT fibers were successfully fabricated from reclaiming the iron particles by the FCCVD method. After CVD tail gas was introduced into the rear furnace, the precursor was injected into the reactor along with deionized water and hydrogen. After a while, threads assembly was stacked at the end of the reactor, which could spin approximately $20 \mathrm{~cm}$ long RCNT fibers. Finite threads integrate was assembled instead of continuous sock-like integrate due to inadequate quantity of CNTs. The yield ratio of fibers to carbon source is shown to the utilization rate of carbon, the yield ratio of RCNT fibers to the carbon source was 0.9 wt.\%, lower than the 4 wt. $\%$ FCNT fibers' yield ratio. The limited amount of deactivated iron nanoparticles leads to the utilization rate reducing.
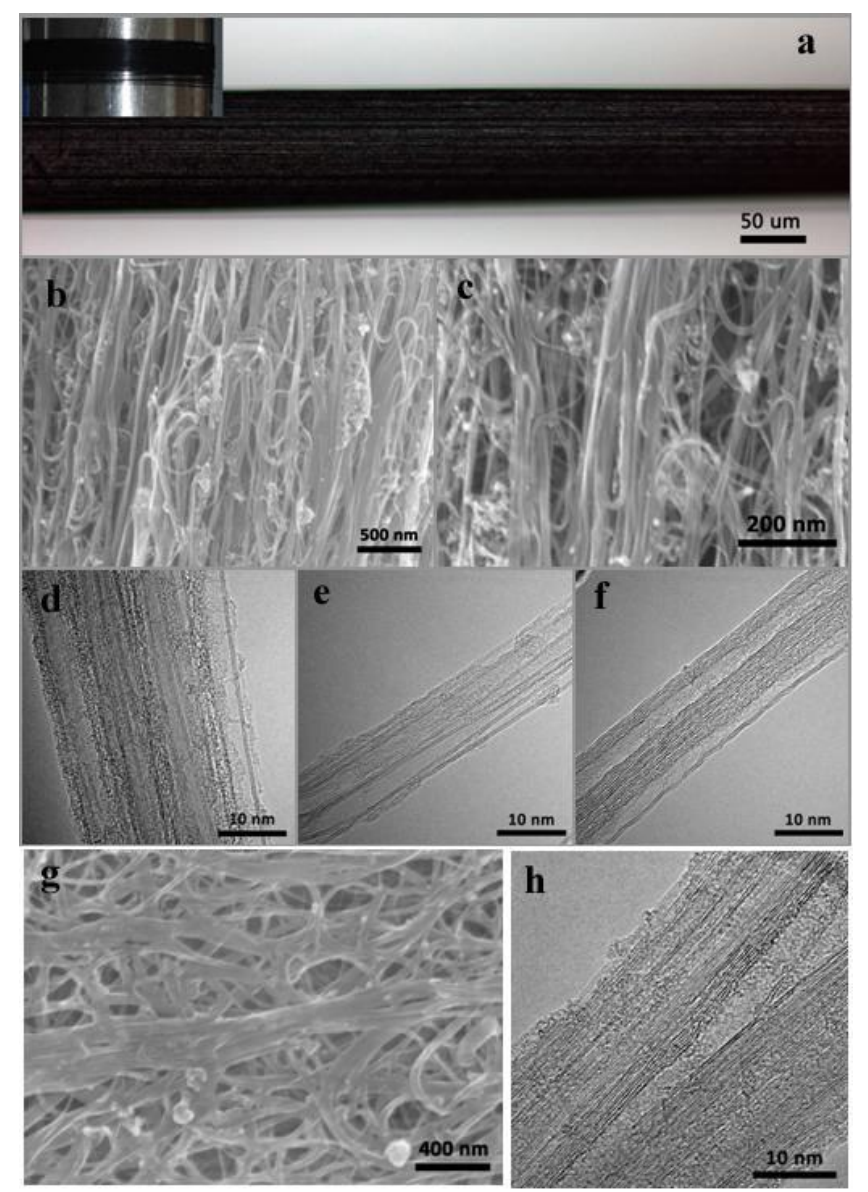

Fig. 3. Morphology and structure of the CNT fiber synthesized by double furnaces system: a-an optical microscope image showing surface of the RCNT yarn; $b-c$ SEM images showing aligned nanotube bundles of the RCNT yarn; $d-$ f TEM images showing CNT bundles and DWNT structure of the RCNT yarn; g-SEM image showing nanotube bundles of the FCNT yarn; $\mathrm{h}$-TEM image showing CNT bundles and DWNT structure of the FCNT yarn

The RCNT fibers were uniform and compact (Fig. 3 a). The SEM images show the distribution of CNT bundles, in which connected nanotube bundles align along the yarn axis, these bundles are loose and thin, with diameters in the range of 20 to $50 \mathrm{~nm}$ (Fig. $3 \mathrm{~b}$ to c). CNTs assembled to form bundles by the van der Waals force. Cotton-like clusters also formed, which blocked CNT bundle assembly. TEM observations indicate that the fiber is mainly composed of DWNTs. Isolated SWNTs can also be observed, with diameters of $3 \mathrm{~nm}$ (Fig. $3 \mathrm{~d}-\mathrm{f}$ ). Iron nanoparticles can be observed in CNT bundles, these iron particles are packed by CNTs, with diameters ranging from 3 to $5 \mathrm{~nm}$. The morphology and structure of FCNT yarns were also researched (Fig. $3 \mathrm{~g}-\mathrm{h}$ ), there is no difference between RCNT fibers and FCNT fibers.
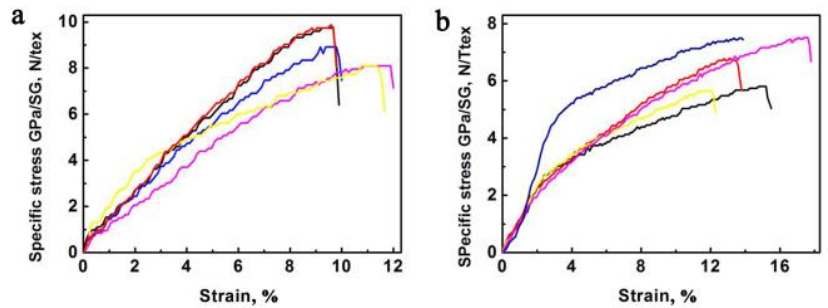

Fig. 4. $a$-the stretch curve of the RCNT fibers prepared by reclaiming the iron nanoparticles in tail gas; $b-$ the stretch curve of the FCNT fibers

The linear density of as-spun RCNT fiber is $0.18 \mathrm{~g} \mathrm{~cm}^{-1}$ (tex), measured by weighing $1 \mathrm{~m}$ fiber with a five-digit scale. The average stress of the fibers is $16.9 \mathrm{cN}$, and the strength of the fibers is $1.6 \mathrm{GPa}$ (Fig. 4 a). The strength of FCNT was also studied, and it was found to reach to $1.27 \mathrm{GPa}$ (Fig. 4 b). The RCNT fibers obtained by reclaiming iron nanoparticles in tail gas have low density and high strength.

The ideal electrical conductivities of RCNT and FCNT fibers are $4.7 \times 10^{3} \mathrm{~S} \mathrm{~m}^{-1}$ and $5.3 \times 10^{5} \mathrm{~S} \mathrm{~m}^{-1}$, respectively, as measured by two-point analysis. The loose nanotube bundles cause the decrease in electrical conductivity by decreasing electron transmission in CNT yarns [35].

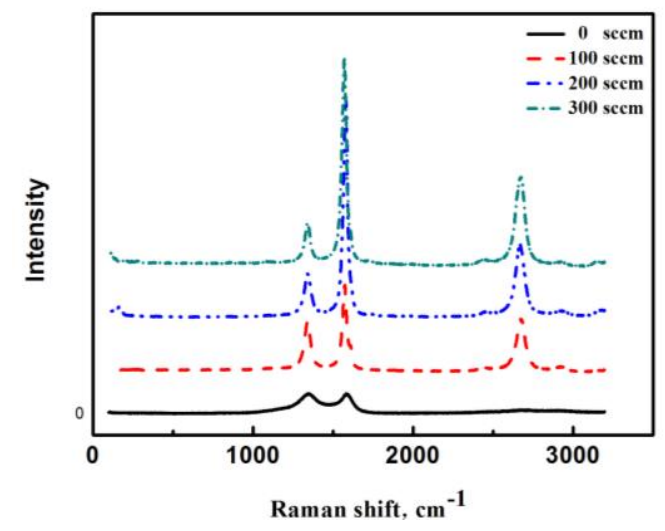

Fig. 5. Raman spectra of the RCNT fibers synthesized with different amounts of hydrogen

During the production process, if hydrogen was stopped injecting into the rear furnace, a small amount of black smoke dispersed in the rear reactor with the aerogel and assembly. The crystalline level of CNT fibers with different amounts of hydrogen was analyzed. The $\mathrm{G}$ peak indicates features of graphite, but the D peak suggests disordered features of graphitic sheet. The ratio of the fiber's $D$ to $G$ peaks indicates the crystalline level of fiber $[36,37]$. As shown in Fig. 5, the crystalline level increases as the hydrogen amount increases. However, too much hydrogen is disadvantage to synthesis CNT assemble, because the concentration of carbon and catalyst decreases. This finding proves that hydrogen is beneficial for keeping 
catalyst activity and increasing carbon transformation to CNT, because hydrogen can remove deposited carbon from the catalyst surface and prevent nanoparticle oxidation [38].

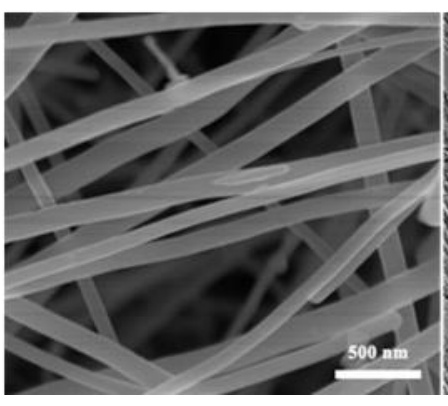

a

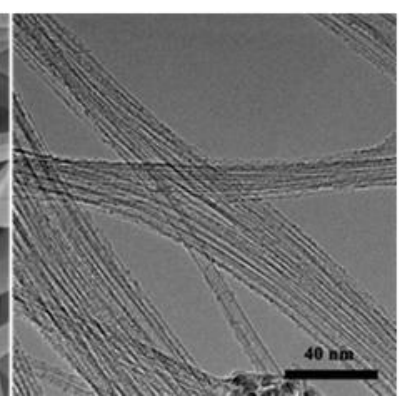

b
Fig. 6. Structure of the assembly synthesized without injecting deionized waterL: a-SEM image showing nanotube bundles of the assemble; $b$-TEM image showing the CNT structure of the assembly

If water vapour injection into the rear furnace ceased, only a small assembly was stacked at the end of the rear reactor. The assembly was collected and analysed, it was found to be composed of CNT bundles. The diameter of the bundles is in the range of 100 to $300 \mathrm{~nm}$ (Fig. 6 a), and the bundles were composed of DWNTs and MWNTs (Fig. 6 b). In these bundles, a small amount of amorphous carbon is packed by CNTs. It indicates that water vapor is beneficial for activating the iron particles and maintaining catalyst activity because can react with amorphous carbon at high temperature and keep the catalyst particles clean [21].

Furthermore, if hydrocarbon feedstock was stopped injecting into the rear furnace, only tail gas, hydrogen, and water vapor flowed through the reactor, nothing happed at all. It proves that the CNT assembly did not result from tail gas. Additionally, if only FCCVD tail gas was stopped injecting into the rear furnace, the assembly would disappear and only smoke would disperse in the reactor. The smock was assumed to be decomposed by the carbon source. If FCCVD tail gas was freshly injected, the threads assembly would begin to stack at the bottom of the reactor again. These findings demonstrate that the deactivated iron nanoparticles in tail gas can be activated to promote the nucleation and growth of CNT.

The deactivated iron nanoparticles in the FCCVD tail gas were reclaimed successfully to promote the synthesis of CNT fibers. The particles, which were wrapped in amorphous carbon layer, were injected into the rear reactor with the gas flow. Then, the deactivated iron particles were activated under the effect of hydrogen and water vapour, which reacted with the amorphous carbon layer and maintained the catalyst activity at high temperature. Besides, bits of unused iron nanoparticles may also exist in tail gas and discharge into the rear furnace with the tail gas.

The morphology of iron particles in RCNT fibers was also analyzed and compared with that of iron particles in FCNT fibers (Fig. $7 \mathrm{a}-\mathrm{d}$ ). The morphology of the particles did not change, but the number of iron particles in RCNT fibers obviously decreased. The size distribution of the iron particles was also more concentrated, with the range of 3 to $7 \mathrm{~nm}$ according to the statistical analysis. The interaction among CNTs as the number of iron particles in the CNT bundles decreased, which contributed to improving the strength of the CNT fibers.
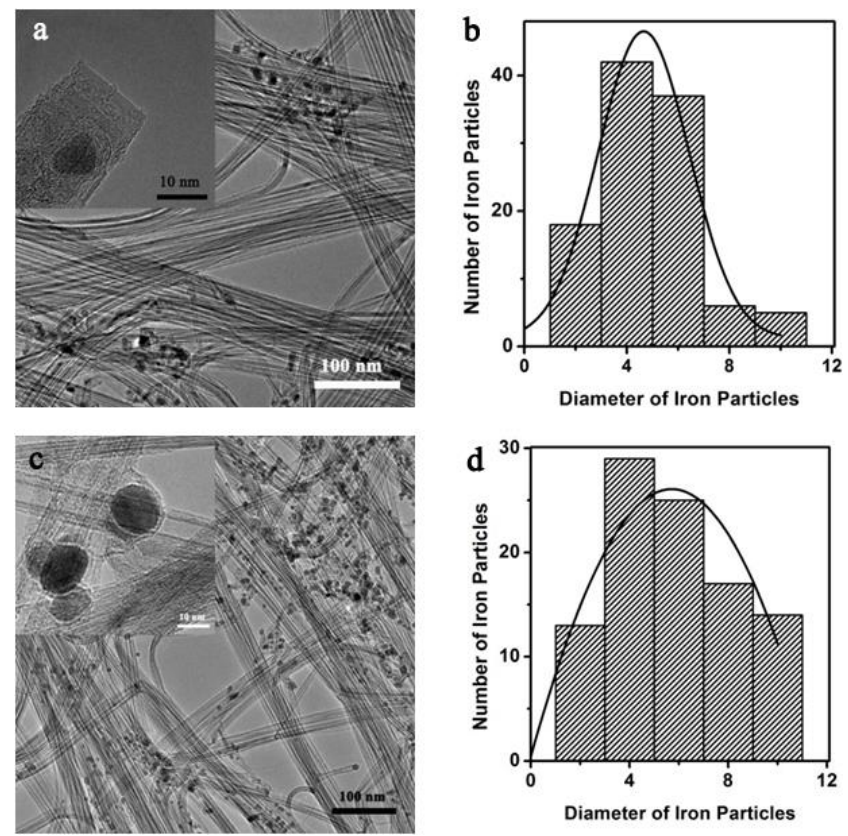

Fig. 7. The morphology of iron particles in CNT fibers: a-TEM image showing the iron particles in the RCNT fibers; $b$-the size distribution of iron nanoparticles in RCNT fibers; $c$-TEM image showing the iron particles in FCNT fibers; $d$-the size distribution of iron nanoparticles in FCNT fibers

The RCNT fibers are compared with the FCNT fibers. Notably, the structure and properties of fibers fabricated in tail gas did not change, and the morphology of the deactivated iron particles and mixed tail gas did not affect the CNT fibers. This finding indicates that it is feasible to synthesize CNT fibers by reclaiming tail gas and activating the deactivated iron particles. The RCNT fibers have excellent properties and reclaiming tail gas can promote the clean and cheap production of CNT fibers.

Moreover, this research also proves that spinning CNT fibers by reclaiming CVD tail gas is feasible in a double furnaces system. The double furnace system not only recycles tail gas but also promotes activation of the deactivated iron particles. In addition, the rear furnace can continuously spin CNT fibers by injecting the precursor with the catalyst into the tail gas. The double furnace system promotes the large-scale and clean production of CNT fibers.

\section{CONCLUSIONS}

FCCVD tail gas contains deactivated iron nanoparticles, hydrogen and carbide gas which includes methane, carbon monoxide, and carbon dioxide. Research shows that the deactivated iron nanoparticles in tail gas can be activated after reacting with hydrogen and water vapor at high temperature under certain conditions. High performance CNT fibers can be successfully prepared by activating the deactivated iron nanoparticles and 
reclaiming tail gas with the double furnaces system. The RCNT fibers produced by this system are composed of DWNTs and a few SWNTs with low density, high strength, and high electrical conductivity. The FCNT fibers have the same structure as the RCNT fibers, and they exhibit excellent properties. These findings indicate that synthesizing CNT fibers by activating the deactivated iron particles and reclaiming tail gas is feasible. This research also proves that spinning CNT fibers in double furnaces system is feasible. The double furnace system promotes the large-scale and clean production of CNT fibers. This research is also beneficial for saving resources and protecting the environment.

\section{Acknowledgement}

This work is supported by national natural science foundation of china (51272172), fund of key laboratory of advanced ceramics and machining technology (Tianjin University) ministry of education (ACMT-2008-03).

\section{REFERENCE}

1. Dai, H. Carbon Nanotubes: Synthesis, Integration, and Properties Accounts of Chemical Research 35 (12) 2002: pp. $1035-1044$.

https://doi.org/10.1021/ar0101640

2. Dresselhaus, M.S., Dresselhaus, G., Saito, R. Physics of Carbon Nanotubes Carbon 33 (7) 1995: pp. 883-891.

3. Niyogi, S., Hamon, M.A., Hu, H., Zhao, B., Bhowmik, P., Sen, R., Itkis, M.E., Haddon, R.C. Chemistry of SingleWalled Carbon Nanotubes Accounts of Chemical Research 35 (12) 2002: pp. 1105-1113.

https://doi.org/10.1021/ar010155r

4. Ajayan, P.M., Tour, J.M. Materials Science: Nanotube Composites Nature 447 (7148) 2007: pp. 1066-1068.

5. Calvert, P. Nanotube Composites - a Recipe for Strength Nature 399 (6733) 1999: pp. 210-211.

6. Kuzhir, P., Paddubskaya, A., Bychanok, D., Nemilentsau, A., Shuba, M., Plusch, A., Maksimenko, S., Bellucci, S., $\quad$ Coderoni, L., $\quad$ Micciulia, F., Sacco, I., Rinaldi, G., Macutkevic, J., Seliuta, D., Valusis, G., Banys, J. Microwave Probing of Nanocarbon Based Epoxy Resin Composite Films: Toward Electromagnetic Shielding Thin Solid Films 519 2011: pp. 4114-4118.

https://doi.org/10.1016/j.tsf.2011.01.198

7. Kuzhir, P.P., Paddubskaya, A.G., Shuba, M.V., Maksimenko, S.A., Celzard, A., Fierro, V., AmaralRabat, G., Pizzi, A., $\quad$ Valusis, G., Macutkevic, J., Ivanov, M., Banys, J., Bistarrelli, S., Cataldo, A. Electromagnetic Shielding Efficiency in Ka-band: Carbon Foam Versus Epoxy/Carbon Nanotube Composites Journal of Nanophotonics 6 2012: pp. 1-18. https://doi.org/10.1117/1.JNP.6.061715

8. Lin, L., Liu, S., Fu, S., Zhang, S., Deng, H., Fu, Q. Fabrication of Highly Stretchable Conductors Via Morphological Control of Carbon Nanotube Network Small 9 (21) 2013: pp. 3620-3629.

9. Liu, K., Sun, Y., Liu, P., Lin, X., Fan, S., Jiang, K. CrossStacked Superaligned Carbon Nanotube Films for Transparent and Stretchable Conductors Advanced Functional Materials 21 (14) 2011: pp. 2721-2728.

10. Feng, Y., Lee, K., Farhat, H., Kong, J. Current on/off Ratio Enhancement of Field Effect Transistor with Bundled
Carbon Nanotubes Journal of Applied Physics 106 (10) 2009: pp. $1-5$.

11. Li, W.Z., Liang, C.H., Zhou, W.J., Qiu, J.S., Zhou, Z.H., Sun, G.Q., Xin, Q. Preparation and Characterization of Multiwalled Carbon Nanotube-Supported Platinum for Cathode Catalysts of Direct Methanol Fuel Cells Journal of Physical Chemistry B 107 (26) 2003: pp. 6292-6299.

12. Serp, P., Corrias, M., Kalck, P. Carbon Nanotubes and Nanofibers in Catalysis Applied Catalysis a-General 253 (2) 2003: pp. $337-358$.

13. Ghosh, S., Sood, A.K., Kumar, N. Carbon Nanotube Flow Sensors Science 299 (5609) 2003: pp. 1042-1044.

14. Thostenson, E.T., Chou, T.W. Carbon Nanotube Networks: Sensing of Distributed Strain and Damage for Life Prediction and Self Healing Advanced Materials 18 (21) 2006: pp. $2837-2841$.

15. De Volder, M.F., Tawfick, S.H., Baughman, R.H., Hart, A.J. Carbon Nanotubes: Present and Future Commercial Applications Science 339 (6119) 2013: pp. 535-539.

16. Behabtu, N., Young, C.C., Tsentalovich, D.E., Kleinerman, O., Wang, X., Ma, A.W., Bengio, E.A., Ter Waarbeek, R.F., De Jong, J.J., Hoogerwerf, R.E., Fairchild, S.B., Ferguson, J.B., Maruyama, B., Kono, J., Talmon, Y., Cohen, Y., Otto, M.J., Pasquali, M. Strong, Light, Multifunctional Fibers of Carbon Nanotubes with Ultrahigh Conductivity Science 339 (6116) 2013: pp. $182-186$

17. Davis, V.A., Parra-Vasquez, A.N., Green, M.J., Rai, P.K., Behabtu, N., Prieto, V., Booker, R.D., Schmidt, J., Kesselman, E., Zhou, W., Fan, H., Adams, W.W., Hauge, R.H., Fischer, J.E., Cohen, Y., Talmon, Y., Smalley, R.E., Pasquali, M. True Solutions of SingleWalled Carbon Nanotubes for Assembly into Macroscopic Materials Nature Nanotechnology 4 (12) 2009: pp. $830-834$

18. Ericson, L.M., Fan, H., Peng, H., Davis, V.A., Zhou, W., Sulpizio, J., Wang, Y., Booker, R., Vavro, J., Guthy, C., Parra-Vasquez, A.N., Kim, M.J., Ramesh, S., Saini, R.K., Kittrell, C., Lavin, G., Schmidt, H., Adams, W.W., Billups, W.E., Pasquali, M., Hwang, W.F., Hauge, R.H., Fischer, J.E., Smalley, R.E. Macroscopic, Neat, SingleWalled Carbon Nanotube Fibers Science 305 (5689) 2004: pp. $1447-1450$.

19. Vigolo, B., Penicaud, A., Coulon, C., Sauder, C., Pailler, R., Journet, C., Bernier, P., Poulin, P. Macroscopic Fibers and Ribbons of Oriented Carbon Nanotubes Science 290 (5495) 2000: pp. 1331-1334.

20. Zhang, X.B., Jiang, K.L., Teng, C., Liu, P., Zhang, L., Kong, J., Zhang, T.H., Li, Q.Q., Fan, S.S. Spinning and Processing Continuous Yarns from 4-Inch Wafer Scale Super-Aligned Carbon Nanotube Arrays Advanced Materials 18 (12) 2006: pp. 1505-1510. https://doi.org/10.1002/adma.200502528

21. Li, Q.W., Zhang, X.F., DePaula, R.F., Zheng, L.X., Zhao, Y.H., Stan, L., Holesinger, T.G., Arendt, P.N., Peterson, D.E., Zhu, Y.T. Sustained Growth of Ultralong Carbon Nanotube Arrays for Fiber Spinning Advanced Materials 18 (23) 2006: pp. 3160-3163.

22. Jiang, K., Li, Q., Fan, S. Nanotechnology: Spinning Continuous Carbon Nanotube Yarns Nature 419 (6909) 2002: pp. 801.

23. Zhang, M., Atkinson, K.R., Baughman, R.H. Multifunctional Carbon Nanotube Yarns by Downsizing An Ancient Technology Science 306 (5700) 
2004: pp. $1358-1361$.

24. Ma, W., Liu, L., Yang, R., Zhang, T., Zhang, Z., Song, L., Ren, Y., Shen, J., Niu, Z., Zhou, W., Xie, S. Monitoring a Micromechanical Process in Macroscale Carbon Nanotube Films and Fibers Advanced Materials 21 (5) 2009: pp. $603-608$.

25. Liu, K., Sun, Y., Zhou, R., Zhu, H., Wang, J., Liu, L., Fan, S., Jiang, K. Carbon Nanotube Yarns with High Tensile Strength Made by A Twisting and Shrinking Method Nanotechnology 21 (4) 2010: pp. 45701-45708.

26. Li, Y.L., Kinloch, I.A., Windle, A.H. Direct Spinning of Carbon Nanotube Fibers from Chemical Vapor Deposition Synthesis Science 304 (5668) 2004: pp. 276-278.

27. Koziol, K., Vilatela, J., Moisala, A., Motta, M., Cunniff, P., Sennett, M., Windle, A. Direct Spinning of Carbon Nanotube Fibers from Chemical Vapor Synthesis Science 318 2007: pp. $1892-1895$. https://doi.org/10.1002/adma.200902943

28. Zhong, X.H., Li, Y.L., Liu, Y.K., Qiao, X.H., Feng, Y., Liang, J., Jin, J., Zhu, L., Hou, F., Li, J.Y. Continuous Multilayered Carbon Nanotube Yarns Advanced Materials 22 (6) 2010: pp. 692-696.

29. Wang, J.N., Luo, X.G., Wu, T., Chen, Y. High-Strength Carbon Nanotube Fibre-Like Ribbon with High Ductility and High Electrical Conductivity Nature Communication 5 (3848) 2014: pp. $1-8$.

30. Reguero, V., Alemán, B., Mas, B., Vilatela, J. Controlling Carbon Nanotube Type in Macroscopic Fibers Synthesized by the Direct Spinning Process Chemistry of Materials 26 (11) 2014: pp. 3550-3557.

31. Motta, M., Moisala, A., Kinloch, I.A., Windle, A.H. High Performance Fibres from 'Dog Bone' Carbon Nanotubes Advanced Materials 19 (21) 2007: pp. 3721-3726.
32. Paukner, C., Koziol, K.K. Ultra-Pure Single Wall Carbon Nanotube Fibres Continuously Spun Without Promoter Scientific Reports 4 (3903) 2014: pp. 1-7. https://doi.org/10.1038/srep03903

33. Sundaram, R.M., Koziol, K.K., Windle, A.H. Continuous Direct Spinning of Fibers of Single-Walled Carbon Nanotubes with Metallic Chirality Advanced Materials 23 (43) 2011: pp. 5064-5068.

34. Che, Y., Wang, C., Liu, J., Liu, B., Lin, X., Parker, J., Beasley, C., Wong, H.S., Zhou, C. Selective Synthesis and Device Applications of Semiconducting Single-Walled Carbon Nanotubes using Isopropyl Alcohol as Feedstock ACS Nano 6 (8) 2012: pp. 7454-7462.

35. Chou, T., Wu, A.S. Carbon Nanotube Fibers for Advanced Composites Material Today 15 (7) 2012: pp. 302-310.

36. Jung, Y., Song, J., Huh, W., Cho, D., Jeong, Y. Controlling The Crystalline Quality of Carbon Nanotubes with Processing Parameters from Chemical Vapor Deposition Synthesis Chemical Engineering Journal 228 2013: pp. 1050 - 1056.

37. Jorio, A., Saito, R., Hafner, J.H., Lieber, C.M., Hunter, M., McClure, T., Dresselhaus, G., Dresselhaus, M.S. Structural (n,m) Determination of Isolated Single-Wall Carbon Nanotubes by Resonant Raman Scattering Physical Review Letters 86 (6) 2001: pp. $1118-1121$.

38. Conroy, D., Moisala, A., Cardoso, S., Windle, A., Davidson, J. Carbon Nanotube Reactor: Ferrocene Decomposition, Iron Particle Growth, Nanotube Aggregation and Scale-Up Chemical Engineering Science 65 (10) 2010: pp. $2965-2977$.

https://doi.org/10.1016/j.ces.2010.01.019 